\title{
Sketching to support visual learning with interactive tutorials
}

\author{
Antje Kohnle ${ }^{1,{ }^{*}}$ Shaaron E. Ainsworth $\odot,{ }^{2}$ and Gina Passante ${ }^{3}$ \\ ${ }^{1}$ School of Physics and Astronomy, University of St Andrews, St Andrews KY16 9SS, United Kingdom \\ ${ }^{2}$ Learning Sciences Research Institute, School of Education, University of Nottingham, \\ Nottingham NG8 1BB, United Kingdom \\ ${ }^{3}$ Department of Physics, California State University Fullerton, Fullerton, California 92831, USA
}

(Received 28 June 2019; accepted 20 December 2019; published 4 December 2020)

\begin{abstract}
[This paper is part of the Focused Collection on Curriculum Development: Theory into Design.] This manuscript discusses how learning theories have been applied to shape multiple aspects of the design of curricular activities combining interactive computer simulations and University of Washington style tutorials (so-called simulation-tutorials). When considering the curriculum goals (what to teach), we drew on theories of representational competence and learning with multiple representations. When considering how to teach, we drew on theories of constructivism and sketching to learn, leveraging the advantages of sketching as a constructive process that requires students to make their current understanding explicit in visual form, to make specific choices in order to make their ideas concrete, and to organize information to support deep processing. When considering when and why to sketch, we drew upon theories of representational competence, learning with multiple representations and inventing to prepare for future learning to describe six distinct purposes of sketching both prior to and while working with the simulation. This is illustrated by presenting specific sketching tasks to show how theory informed the design and the sequencing of the tasks. We followed a design-based research method, working at two institutions in two countries and with multiple cohorts of students to understand, and where necessary improve, the design of these activities, primarily basing our decisions on the sketches that students had created. The key message of this research is that the design and sequencing of sketching tasks needs to be carefully matched to the pedagogical rationale and that theory can shape these decisions in many ways.
\end{abstract}

DOI: 10.1103/PhysRevPhysEducRes.16.020139

\section{INTRODUCTION}

Expert physicists use representations such as equations, graphs, and diagrams to reason about phenomena, imagine new situations, test ideas, and explain their findings to peers, students, and the general public (e.g., Refs. [1,2]). They know how such representations work, how they relate to one another, and how to select an appropriate representation for the task at hand. Based upon their experimental and ethnographic studies with chemists, Kozma and Russell termed these skills and practices "representational competence" [3]. Since then, other researchers have documented how representational competence is not limited to chemistry but is found across the science, technology, engineering, and mathematics disciplines, for example, in biology [4,5], geology [6], mathematics [7,8], and physics [9-12].

*ak81@st-andrews.ac.uk

Published by the American Physical Society under the terms of the Creative Commons Attribution 4.0 International license. Further distribution of this work must maintain attribution to the author(s) and the published article's title, journal citation, and DOI.
Various authors have pointed out the role of representational competence in physics in terms of problem-solving ability, deep understanding, emergent insights, and transfer of knowledge across contexts [9,13-17]. Students need to be able link multiple representations to learn from physics texts and communicate physics ideas [18]. And, although representational competence is fundamental to professional practice across the scientific disciplines, abundant research has shown that it is very demanding for students to acquire (e.g., Refs. $[3,5,19,20])$. For physics in particular, various studies have documented students' difficulties in employing representations correctly and using them consistently and reflectively [21-24].

Kozma and Russell [3] proposed a developmental progression consisting of five levels and used this to explain how people acquire representational competence. At level 1, representations are treated as depictions, perceptually similar to the underlying phenomena. At level 2, this knowledge is supplemented as students recognize some symbolic aspects of representations but they focus on the surface features. Elby [25] refers to this as "what you see is what you get" and provides vivid descriptions of its application; for example, in the classic example of a velocity-time graph of a cyclist going over a hill, where 
some students expect this graph to be hill shaped. Then as students' experience develops, at level 3, they may be able to interpret formal representations but are limited to reasoning about them syntactically and relating them to others in a syntactic fashion (e.g., using surface features rather than their underlying meaning). For example, given a position-time graph and a table of velocity values, students might reason syntactically "the slope of the line is decreasing and so are the numbers in the table." However, when students master semantic use of formal representations (level 4), they relate representations to the underlying meaning of the physical phenomenon they represent. This means they can go beyond syntactic reasoning to think about what such representations mean and relate them through this understanding (e.g., "the graph and the table are both showing decreasing velocity"). At the final stage of representational competence (level 5), people have the abilities to choose and reflect on the most appropriate representation for a given task, and use specific features of representations to justify their claims in a social context. Thus, as experts they have become reflective rhetorical users of the representations of their professional practice with fully developed representational competence.

This manuscript discusses how learning theories have been applied to develop tasks that center on sketching, which we define broadly as the purposeful construction of a visual representation. All the tasks were intended to support visual learning with simulation-tutorials. These simulationtutorial activities combine question sequences in the style of the University of Washington tutorials [26] with interactive computer simulations, and are intentionally constructed to support the development of representational competence in quantum mechanics. The simulation-tutorials were designed for intermediate (junior or senior) level undergraduate quantum mechanics and tested with students at both U.S. and UK universities with different student populations and quantum mechanics curricula $[27,28]$. The activities leverage features of interactive computer simulations, including interactivity and direct feedback, visualizations that can make the invisible visible, the use of multiple representations, and allowing students to quickly change parameters to explore relationships between quantities [29-33]. Our approach is aligned with work in chemistry education that has utilized interactive computer simulations to promote representational competence [19].

We define "visual learning" as the processes of interpreting and constructing visual representations in order to enhance understanding of these representations and the underlying physics concepts and phenomena (i.e., representational competence). Note that here and throughout we use the term "visual representation" to denote diagrams, pictures, and graphs, and "symbolic representation" to denote mathematical (algebraic) expressions. Although our central concern is with visual representations, they do not exist in isolation, and thus we are also concerned with their relationship to other forms of representations, especially symbolic representations. Definitions of representational competence as well as theories of learning and use of multiple representations (e.g., Refs. [18,34]) emphasize that understanding how representations relate to one another is fundamental to their successful use. Moreover, abundant research (e.g., Refs. [16,18,35,36]) shows that knowing how to relate representations can be particularly challenging for students and accordingly, instruction should focus on supporting students in this task (e.g., Ref. [37]).

To advance our understanding of theory and to improve the educational experiences for our students, we adopted a designbased research approach (DBR). DBR aims to design and implement educational experiences for specific contexts and systematically study them to iterate their design and advance understanding of theory [38-40]. Theory, for design-based researchers, is not grand but "humble" in that it addresses domain specific learning processes [41]. Consequently, although it may draw on both grand theories (e.g., constructivism) as well as the more specific, the intended outcome of the research is a theory that explains and improves learning by generating practical design principles for a particular set of concepts and contexts. Thus, our overarching research question was how sketching combined with interactive tutorials can best be designed to support the development of representational competence in quantum mechanics.

This manuscript discusses how learning theories shaped the curriculum goals, the overall pedagogical approach and choices made at the level of activity phases, individual tasks and their sequencing in the simulation-tutorials. To this end, the manuscript is structured as follows: In Sec. II, we show how learning theories shaped what students should come to know (i.e., representational competence in quantum mechanics). In Sec. III, we show how they influenced the overall pedagogical approach in terms of sketching to support learning. In Sec. IV, we show how learning theories shaped when students sketched and why sketching needed to play very specific roles to support learning in the designed activities. In Sec. V we illustrate how students responded to these activities to reflect on our choices and improve them where needed. Section VI returns to explore how these findings shaped our understanding of the roles of theory in our activities.

\section{REPRESENTATIONAL COMPETENCE IN QUANTUM MECHANICS}

The first way that learning theory shaped the design of the activities was in setting out the overall learning goal of representational competence in quantum mechanics. We thus here adopt a domain-specific (i.e., physics subject specific) definition of representational competence $[42,43]$ rather than defining it in the absence of specific content. When we refer to how representations work in general, we instead draw on diSessa's conceptualization of metarepresentational competence [44]. 
A common finding in quantum mechanics is that students can perform calculations but then struggle to interpret and evaluate their results [45]. The resources described in this article aim to help students master visual representations of quantum ideas and reason through them starting from the known mathematical expressions. Our stance is that mastery of both visual and symbolic representations of quantum ideas can reduce incorrect ideas based on algorithmic use of mathematics, allow for multiple approaches (both quantitative and qualitative) to solving a problem, allow checks of consistency to evaluate and interpret outcomes, and support the development of representational competence in terms of expertlike problem solving.

Visual representations in quantum mechanics can pose particular challenges due to the complex nature of the wave function, the inherent uncertainty in some of the quantities that are depicted (such as in the semiclassical vector model), the abstract nature of quantum properties such as spin, largedimensional Hilbert spaces (the difference between real space and Hilbert space), and the potential confusion with similar depictions in classical mechanics [46,47].

Representational competence in quantum mechanics involves the interpretation and construction of canonical visual representations. Both of these aspects are challenging for the learner. For example, the solutions to the onedimensional infinite well and the harmonic oscillator are commonly depicted by showing the potential energy, the first few energy levels, and the associated spatial parts of the energy eigenfunctions all in a single nested diagram (see, e.g., Fig. 1 in Ref. [48]). In these diagrams, the vertical dimension has two different meanings, namely, energy and the amplitude of the wave function. While these diagrams are very efficient, the multitude of features require a high level of representational competence in order to successfully reason with them. This is not a problem for experts, but may require additional instruction for students. As another example, the modulus squared of the spherical harmonics as angular solutions to the hydrogen atom electron are often depicted via three-dimensional graphs. In our experience, it is challenging for students to interpret these graphs correctly in terms of directional probability densities that contain no information on the distance between electron and proton.

An example of the challenges of constructing representations in quantum mechanics and transforming across them is shown in the student work in Fig. 1. The student has correctly stated the mathematical expression for the wave function of an energy eigenstate in an infinite square well. However, when they transformed this symbolic representation to a visual one, they ignored the imaginary component of the wave function, leading to an incorrect visual representation where the wave function does not remain normalized as a function of time. This lack of consistency between representations can indicate that the

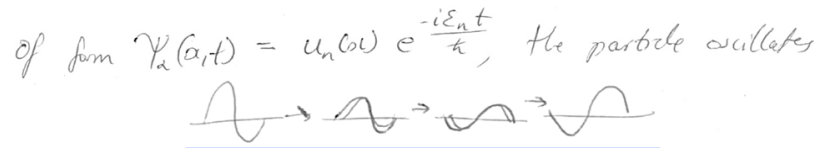

FIG. 1. An example of student work showing inconsistency between representations for the time development of an energy eigenstate in an infinite square well. The student has written the correct mathematical expression, but an incorrect visual depiction.

student was not able to connect them by considering their common underlying physics meaning.

Being able to use visual representations to describe physical systems is an important curriculum goal, and one that students need support to develop. Consequently, we now turn to considering the pedagogical approach that we adopted in an attempt to achieve our ambition.

\section{HOW SKETCHING CAN SUPPORT LEARNING}

The second way that learning theory shaped the design of the activities was in setting out the foundational assumptions that underpin the overall pedagogical approach: in other words, how sketching should be used. Broadly speaking, the approach taken is constructivist, as the intent of the activities is that learners should be active in the construction of their own understanding (e.g., Refs. [49,50]). Specifically, we draw on Chi's definition of a constructive learning activity [51], in which a learner produces an overt output that goes beyond a simple restatement of any studied material in a thoughtful way. Thus, Chi suggests that explaining, elaborating, making predictions, and reflecting are all constructive activities, or have the potential to be if learners do so meaningfully. In our pedagogical design, we focus on how sketching visual representations can be a constructive learning activity.

The last decade has seen an increasing interest in the role of sketching (or drawing) as a way to support students to learn in science. In 2005, Van Meter and Garner found that only a small number of studies explored drawing to learn and that the area had been mostly inactive since the mid 1980s [52]. Since that review (and partly because of that review), the situation has changed markedly. There have been high profile calls for the value of drawing to learn [53], new theories of drawing to learn proposed [54], special editions of journals published [55], and such a large body of new empirical studies that meta-analysis [56] and systematic reviews [57] are now available. Broadly speaking, these accounts align to suggest that sketching can be utilized to be a very effective way to learn in science. Although precise definitions can vary, and some authors use the term sketching, some drawing, and some selfgenerated graphical representations, the essence of the argument is that students benefit by constructing for themselves a representation that is visual in form. Like the researchers cited above, we exclude graphically 
organized text (e.g., mind maps, concept maps) from our definition of sketching; however, some also excluded graphs (e.g., line graphs, histograms) whereas we do not.

Sketching to learn can be used for many different purposes and we describe some of these in detail in Sec. IV. However, the theoretical rationale underlying our general approach is that sketching can be a constructive process that helps learners make their current understandings explicit in visual form. This helps students monitor and reflect on the accuracy of their understanding by activating relevant schemas (knowledge structures), and supports them to overcome any gaps and misconceptions in this understanding. This claim is common to other constructive activities such as generating explanations for others or selfexplanations for oneself (e.g., Ref. [58]). However, in contrast to a written summary or a verbalization as in a self-explanation, sketching results in a permanent, explicit, visual representation. This brings additional benefits.

Visual representations are specific as they require explicit choices to be made [59]. They can augment memory by reducing load on short-term memory so that it can be used more efficiently (e.g., Ref. [60]). Visual representations organize information by grouping together relevant information so that perceptual search is more efficient. Consequently, learners can benefit from more automatic inferences that seem to emerge rather than having to be laboriously constructed [61]. It is these properties of visual representations that have been argued to result in additional or deeper learning, as compared to the learning of scientific concepts through textual representations [62] or verbal self-explanation [63]. Moreover, constructing your own visual representation has been shown to help overcome shallow processing compared with interpreting a given visual representation, where it is possible to skip over or only skim the representation (e.g., Ref. [64]).

\section{WHEN AND WHY TO SKETCH}

The third way that theory influenced our design was in the choice of when and why students sketched. We first focus on when to sketch. In the existing literature on sketching to support visual learning from simulations (which here encompasses animations) learners have been asked to sketch prior to interacting with a simulation to help students prepare to learn, during work with a simulation to deepen their understanding of the representations shown, or after interacting with a simulation to reflect upon this learning (e.g. Refs. [65-67]). Our activities utilized sketching in two of these three phases (see Fig. 2). Students first were asked to work on problems independent of the simulation, constructing visual representations before being presented with visual representations in the simulation (phase 1). They then engaged in new sketching activities while working with the simulation to support their understanding of these representations (phase 2). Reflective sketching after completing work with the simulation was

\section{Phase 1. Without simulation support Students sketch familiar representations and construct/invent unfamiliar representations they later see in the simulation for a particulary simple case.}

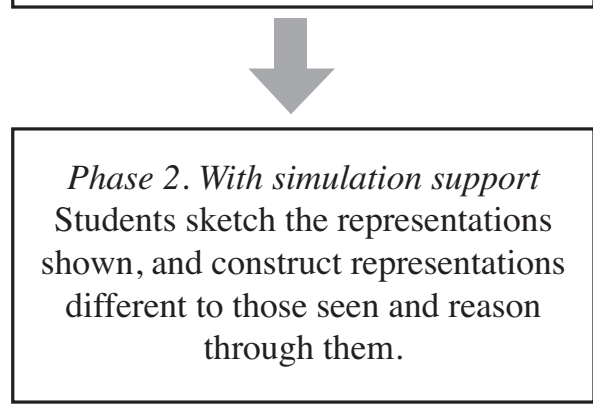

FIG. 2. The two phases of the simulation-tutorial activities discussed in Secs. IVA (phase 1) and IV B (phase 2).

not included due to length and time constraints on the activity.

This two phase approach is aligned with that articulated by Schwartz and colleagues $[68,69]$ whereby students are encouraged to prepare for learning by first working independently to solve problems prior to being exposed to expert solutions. In other words, rather than students being told what to do and then practice it, they attempt parts of the task for themselves first. For example, in inventing to prepare for future learning, (IPFL), Schwartz and Martin [68] describe how IPFL was used with high school students learning statistics. Students first interrogated simple datasets (phase 1), were then given a lecture on representations such as histograms and box and whisker plots, and then practiced what they had now learned. It should be noted that no student was found to have invented a correct solution in phase 1. However, when comparing their performance on subsequent tests to students who had received the traditional lecture and then practice approach, students in the IPFL classes performed equally well on straightforward tests but better on tests where they needed to learn new ideas during the test. Schwartz et al. [69] used a similar approach to teach physics concepts (e.g., density and speed), comparing students who first invented solutions using carefully chosen contrasting cases to students who first were told solutions before studying the contrasting cases, and again found similar benefits. Brahmia et al. developed physics invention tasks in which students generate mathematical quantities prior to formal instruction on these topics [70]. They found substantial gains in conceptual understanding following the implementation of these tasks into introductory physics courses. Thus, we hoped that our two phase approach of asking students to sketch visual representations without the support of the simulation before then sketching again with it should 
provide similar advantages for deeper learning. Our activities intended students to develop an appreciation of the structure of visual representations in quantum mechanics, and thus to be able to transfer their learning to new examples in the future.

The choices of when to sketch in terms of these two phases were crucial to informing why we chose specific sketching tasks and how they were designed to enhance students' learning. Accordingly, we will now describe the theoretical rationale for the specific activities involving sketching in phase 1 (Sec. IVA) and then phase 2 (Sec. IV B). We show that there are distinct roles that sketching serves in both of these phases. We provide examples of tasks that utilize these different roles of sketching from two different simulationtutorial activities in quantum mechanics. Only tasks that include sketching or further roles of sketching are discussed here, but it should be noted these were not the only sorts of task included in the activities. Finally, we will end Sec. IV C by returning to considering when students sketch, but this time in a more fine-grained manner. We consider the role of theory in the sequencing of the specific tasks within each phase.

Two different simulation-tutorials were chosen in Secs. IV A and IV B to show that these roles of sketching apply across multiple quantum mechanics topics implemented in simulations with different visual representations. Consequently, before describing these activities we will first contextualize them by briefly describing the two different simulations.

The two simulation-tutorial activities described below focus on the topics of time development of quantum states and first-order time-independent perturbation theory. The associated simulations are very different in terms of their visual representations. The "time development of infinite well quantum states" simulation [71] includes dynamic, animated graphs (that can be stopped and stepped through) including both the real and complex parts of the wave function in two- and three-dimensional depictions. In contrast, the "energy corrections in a perturbed infinite well" simulation [72] only includes static graphs that are wholly real (not complex) and only show one variable as a function of position as well as an energy level diagram (see Fig. 5). Thus, the visual representations in the time development simulation are more challenging in terms of their inherent dynamical nature, complexity, and dimensionality. However, both simulations include visual representations that are likely to be unfamiliar to students. In the case of time development, this includes the complex depictions of the wave function and for perturbation theory, the product graph of two curves.

\section{A. Sketching to prepare for learning with the simulations}

Sketching was used for three distinct purposes prior to working with the simulation: students were encouraged to sketch to activate their prior knowledge, to transform across representations, and to invent representations. In all of these cases, no visual representation was provided to students. For each purpose, we will now outline the theoretical rationale and then illustrate a specific sketching task to show how theory informed the design.

The sketching tasks discussed in this section are within the simulation-tutorial time development of infinite well quantum states (see Fig. 3). The activity and associated simulation are described in more detail in Ref. [28]. The tasks in Fig. 3 are taken from the beginning of the activity (phase 1 in Fig. 2) before the students have interacted with the simulation. The example sketches in Fig. 3 were purposefully selected to be correct and illustrate important features with respect to the coding scheme discussed in Sec. VA. The learning goals of the activity are for students to develop a visual model for the time evolution of the wave function and how it gives rise to the time evolution (or lack thereof) of the probability density. The first phase of the activity aims to prepare students to understand the visual representations they later see in the simulation by constructing these representations themselves for a particularly simple case (that of the ground-state energy eigenfunction). The focus is on interpreting the time evolution $\psi_{1}(x) e^{-i E_{1} t / \hbar}$

\section{Abbreviated Text}

Example Sketches

A Sketch the ground state energy eigenfunction at $t=0$.

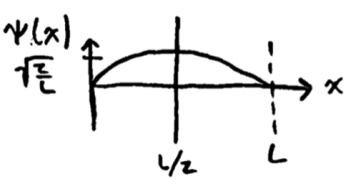

B Plot the time evolution of $\psi_{1}(x, t)$ at $x=L / 2$.

Describe this evolution in words. (Students were given the labeled axes and four times at which to evaluate the function.)

C Sketch $\psi_{1}(x, t)$ at time $E_{1} t / \hbar=3 \pi / 2$
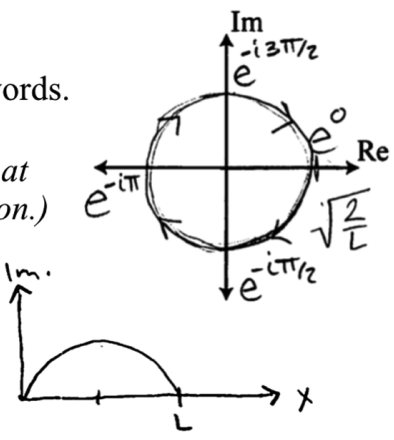

D How would you plot the time evolution for the entire function using a 3D representation?

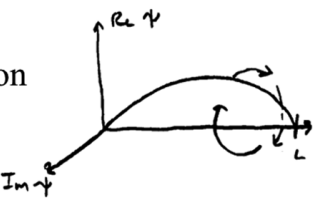

FIG. 3. Abbreviated versions of tasks A, B, C, and D of the time development of infinite well quantum states activity and sample student sketches. They are a subset of the tasks that are asked of students prior to working with the simulation (see Supplemental Material [73] for the full activity). The roles of sketching in these tasks are to activate prior knowledge (task A), to transform across representations (tasks $\mathrm{B}$ and $\mathrm{C}$ ), and to invent representations (task D). 
visually as a rotation of the $\psi_{1}(x)$ curve in the complex plane.

Students will interact with the associated simulation [71] following these tasks. The simulation depicts a wave function at a single point $\psi\left(x_{0}, t\right)$ in the complex plane (Argand diagram) and for all points as a 3D graph of $\psi(x, t)$ with axes depicting the real and imaginary parts of $\psi$ as a function of $x$. It also shows the associated probability density $|\psi(x, t)|^{2}$. Students can choose to display the ground state, the first-excited state, and a superposition of these states.

\section{Sketching to activate prior knowledge}

The first sketching task to help students prepare to learn from the simulations (task A in Fig. 3) was designed to activate their relevant prior knowledge. There is abundant evidence that learning can be enhanced when students are asked to retrieve already known material prior to engaging with learning new content (e.g., Refs. [74-76]). These authors (and others) argue that by doing so a learner can more easily establish connections between their existing knowledge and the new content, thus enhancing understanding and recall. A large number of techniques or strategies have been utilized for this purpose, including simply asking learners to bring to mind their relevant prior knowledge, discussion in small groups, being asked to adopt a particular perspective, or answering exploratory tasks. Of particular relevance to our work are those studies that have found that using visual representations to activate prior knowledge is effective (see Ref. [77] for a review), especially those where learners construct their own representations. Wetzels et al. conclude that this is effective, especially when students have appropriate prior knowledge to draw upon [77]. Moreover, as the activation of prior knowledge will result in an external, inspectable, visual representation, students will be able to benefit from the properties of visual representations articulated in Sec. III.

Task A in Fig. 3 is the first task in the activity and asks students to sketch the ground state energy eigenfunction at time $t=0$. This is a familiar graph that students have seen several times in both the lectures and their textbook, and one that they should know from memory. In addition to activating the prior knowledge required in subsequent tasks, this task also primes students for the activity in that sketching will play a more important role than mathematics in this activity.

\section{Sketching to transform across representations}

In the next sketching tasks (tasks B and C in Fig. 3), students were encouraged to construct a second representation on the basis of an existing one. For example, they were asked to sketch a graph given a mathematical expression or to describe their sketch in words. The rationale underlying these activities is that students benefit from actively working with multiple representations. By doing so, students can develop deeper understanding of phenomena, see the relationships between ideas, create more abstracted understanding that supports generalization, and develop their understanding of the purposes of representations (e.g., Refs. [34,78-80]). Thus, students need to actively integrate representations to develop their representational competence.

Although many of these benefits have been found when students relate two given representations, this is notoriously difficult for students and many simply avoid doing so, working solely with one representation (see Ref. [18] for a review of studies). One solution to this dilemma is to automatically link representations, so that actions on one representation are automatically reflected on a second one (e.g., Ref. [34]). An example would be a user editing values in a table and a corresponding graph being updated automatically. This is the approach taken with the interactive simulation in phase 2 of the activity (see Fig. 2). However, while this may reduce the difficulty of working with multiple representations, it is, of course, only possible when the representations are digital. However, a more general concern is that without appropriate support this approach may leave students too passive, not encouraging deeper processing of the relation between representations. When students need to generate one representation from another, it is a more constructive activity. Hence, the key requirement of the design for phase 1 is that students should be able to achieve this difficult task. Therefore, the examples with which they work should be relatively simple.

There are two transformation tasks in this phase of the activity that use the ground state wave function as a particularly simple case. First, Task B in Fig. 3 asks students to transform from a symbolic to a visual representation, and then from the visual representation to words. Students are asked to take the value of the wave function at a single point (the center of the well), and sketch how the wave function at this point evolves with time. We anticipated that this task would be challenging. Thus, to help students with this task, they are provided with the axes for a complex plane graph and four specific times at which to evaluate the temporal part $e^{-i E_{1} t / \hbar}$ of the wave function (giving values of $1,-i,-1$, and $i$, respectively).

Second, task C in Fig. 3 has students sketch the entire wave function at of one these times, namely a time when the function is completely imaginary, giving $i \psi_{1}(x)$. This is again a transformation from a symbolic representation to a visual representation. The result is a sketch with a very familiar shape (the same shape as in task A), but with a different quantity along the vertical axis.

Within the context of the activity, these tasks serve to prepare students for the visualization they will see in the simulation and for the invention of a 3D representation of the ground state wave function (task D).

\section{Sketching to invent representations}

In the final sketching task in this phase (task D in Fig. 3), students were asked to sketch a representation they have 
not previously seen. We did not want them necessarily to recreate a textbook representation but instead to think about a phenomenon and then invent a visual representation that encapsulates its key components. When completing this task, students utilize both their knowledge of representational forms in quantum mechanics and their metarepresentational competencies, e.g., in terms of designing representations that should be complete, parsimonious, and systematic [44].

The design and placement of our invention task follows the IPFL approach of Schwartz (e.g., Refs. $[68,69])$ in that it occurs prior to exposure to expert canonical representations in the simulation. As argued in Sec. IV, in the design of such tasks, there is no necessity to ensure students invent a correct representation. The key to success seems to be that after attempting to generate a solution for themselves, students are given feedback about correct solutions (e.g., Ref. [81]), perhaps even more so when this solution is unexpected [82]. Thus, in our activity design, it was important that when subsequently working with the simulation in phase 2 , students will be interacting with a correct representation, which they are encouraged to actively process.

In task D in Fig. 3, students are asked how they might plot the entire wave function at all times. Up to this point, they have sketched the entire wave function at two different times (one where it was entirely real and one where it was entirely imaginary), as well as a sketch of how a single point on the function evolves with time. Students are now tasked with how they might include all of this information in a single sketch. As mentioned above, it is not expected (or even desired) that students discover the canonical visualization that they will see in the simulation. This task was designed such that students would have to deeply consider all the information they have worked with thus far and attempt to integrate all the pieces. This task also prepares them to better understand the visualization they will interact with in the simulation.

\section{B. Sketching while learning with the simulations}

We now turn to our considerations of how sketching was used when students were learning with interactive simulations, i.e., with multiple external representations. Again, sketching was used for three distinct purposes: students were encouraged to sketch to support observation, to understand the properties of a visual representation, and to support reasoning. For each role, we again outline the theoretical rationale and then illustrate a specific sketching task to show how theory informed the design.

This section discusses the simulation-tutorial activity energy corrections in a perturbed infinite well (see Fig. 4). Note that this is a different simulation-tutorial activity to that from Sec. IV A. The activity and associated simulation are described in more detail in Ref. [27]. The tasks in Fig. 4 are the first four tasks of the second phase of the activity with simulation support (phase 2 in Fig. 2). The final two tasks of the activity are not discussed here as they do not include either sketching or new roles of sketching. The example sketches in Fig. 4 were purposefully selected to be correct and illustrate important features with respect to the coding scheme discussed in Sec. VA. The screen shot in Fig. 5 shows the setup of the simulation that students use for task E in Fig. 4.

The activity develops a visual understanding of the firstorder time-independent energy corrections $E_{n}^{(1)}$ to the $n$th energy eigenstate for an infinite square well, where the superscript "(1)" stands for first-order correction. For the cases shown in the simulation where the perturbations to the infinite well are functions $V(x)$, the first-order energy correction of the $n$th eigenstate is given by the integral of the perturbation and the corresponding probability density, $E_{n}^{(1)}=\int_{0}^{L} V(x)\left|\psi_{n}(x)\right|^{2} d x$. The learning goals of the activity are for students to be able to reason visually about the sign and relative magnitude of the energy corrections by considering the shape and symmetry of the $V(x)\left|\psi_{n}(x)\right|^{2}$ product curve.

A screen shot of the associated simulation [72] is shown in Fig. 5. The left-hand panel depicts an energy level diagram, with the unperturbed (dashed lines) and perturbed (solid lines) energies displayed. Students can choose different perturbations and set their strength via the middle "main controls" panel. The right-hand panel shows the potential energy $V(x)$, the probability density $\left|\psi_{n}(x)\right|^{2}$ corresponding to the selected energy level and the resulting product graph. The top right panel gives the formula for the energy correction and its sign for the current configuration.

\section{Sketching to observe}

The first sketching task with the simulation (task E in Fig. 4) focused on observational sketching. The purpose of this task was for students to recreate for themselves without further elaboration a visual representation that they were shown. Thus, the sketch does not contain ideas that go beyond the visual information shown. It is simply intended to overcome passive processing of visual representations to ensure that students are more active. When students sketch something they observe, it helps them attend to details and subtle properties that might otherwise not be observed [52]. Sketching has been found to help students overcome the "seductive details" effects of visual representations that can result in shallow processing (e.g., Ref. [83]). Finally, when a student sketches their own copy of a graph on a screen, they then have a permanent record of the representation to consult, and the act of copying it will likely aid memory retention [84].

Task E in Fig. 4 asks students to sketch all of the visual representations shown in Fig. 5 without any transformation or elaboration. This task aims for students to observe more closely the visual representations shown and their features. 
E Consider the perturbation 1 with a positive slider value and the perturbed ground state of the infinite well.

Sketch the unperturbed and perturbed ground state energies as shown in the left-hand diagram in the simulation.

Sketch the right-hand graphs of $V,\left|\psi_{1}\right|^{2}$, and $V\left|\psi_{1}\right|^{2}$

F $\quad$ Sketch the product curve $f(x) g(x)$ Would the inner product $\langle f \mid g\rangle$ be positive, negative, or zero?
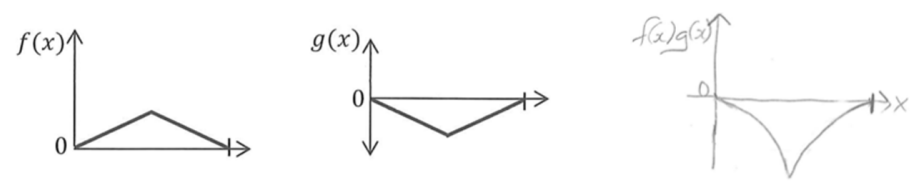

G (i) Which perturbations from the simulation have a zero energy correction for all energy eigenfunctions?

(ii) Sketch a perturbation different from the ones in the simulation (this includes negative slider values) that would have a zero energy correction for all energy eigenfunctions. Explain your reasoning.

$\mathrm{H} \quad$ Two students are discussing the following perturbation $\uparrow$ (not shown in the simulation).

Consider the two statements:

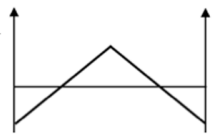

A: "V has equal areas above and below the $x$-axis. This implies that all energy corrections will be zero, as the average value of $V$ is zero. The symmetry of V plays no role."

B: "But isn't the formula $E_{n}^{(1)}=\int V\left|\psi_{n}\right|^{2} d x$, so that we need to consider the product of $V$ and $\left|\psi_{n}\right|^{2}$ ?For the ground state, the probability density is greater in the middle than at the edges, so the product graph won't average to zero."

Draw graphs in order to determine which student is correct. Correct the student's statement.
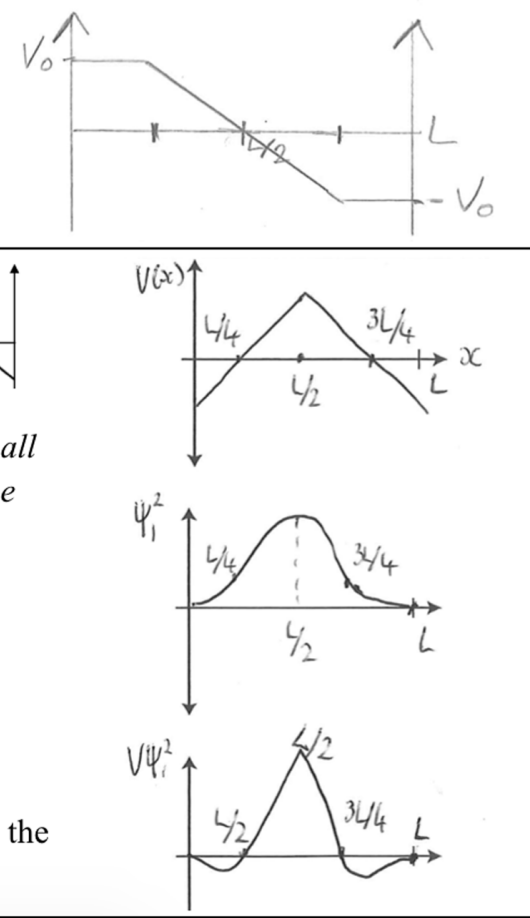

FIG. 4. Abbreviated versions of tasks E, F, G, and H of the energy corrections in a perturbed infinite well activity and sample student sketches. They are only a subset of the tasks that are asked of students while working with the simulation (see Supplemental Material [73] for the full activity). The roles of sketching in these tasks are to observe (task E), to understand the properties of a visual representation (task F), and to reason (tasks $\mathrm{G}$ and $\mathrm{H}$ ). Tasks $\mathrm{E}$ and $\mathrm{H}$ provides three blank axes for students to use that scaffold the sketching of graphs of the perturbation, the probability density, and their product.

These features include the unperturbed and perturbed energy levels and their labels in the energy level diagram (left-hand panel in Fig. 5), and the shapes and axis labels of the three graphs of the perturbation, probability density and product curve (right-hand panel in Fig. 5). Sketching the product graph of the perturbation and probability density in particular requires students to note where the function is zero and where it is maximal. Sketching all three graphs on individual axes requires students to match up particular points, e.g., locations of minima. In order to ensure that students sketch the energy level diagram and all three graphs shown in the simulation, the task has additional scaffolding (not shown in Fig. 4) of an empty energy level diagram and three empty sets of axes without any labels.

\section{Sketching to understand the properties of a visual representation}

The next sketching task (task F in Fig. 4) involved constructing a representation that students had seen and sketched previously, but one that was different in terms of the individual underlying features. The aim of this sketching task was to help students master the properties of a canonical representation, as understanding how such representations encode and present information is a key literacy of science (e.g., Refs. [3,85]). This task was included as abundant research suggests that students can lack this representational knowledge (e.g., Refs. [18,86]), and yet it is clearly a prerequisite to be able to reason with the representations. In the case discussed here, this form of 


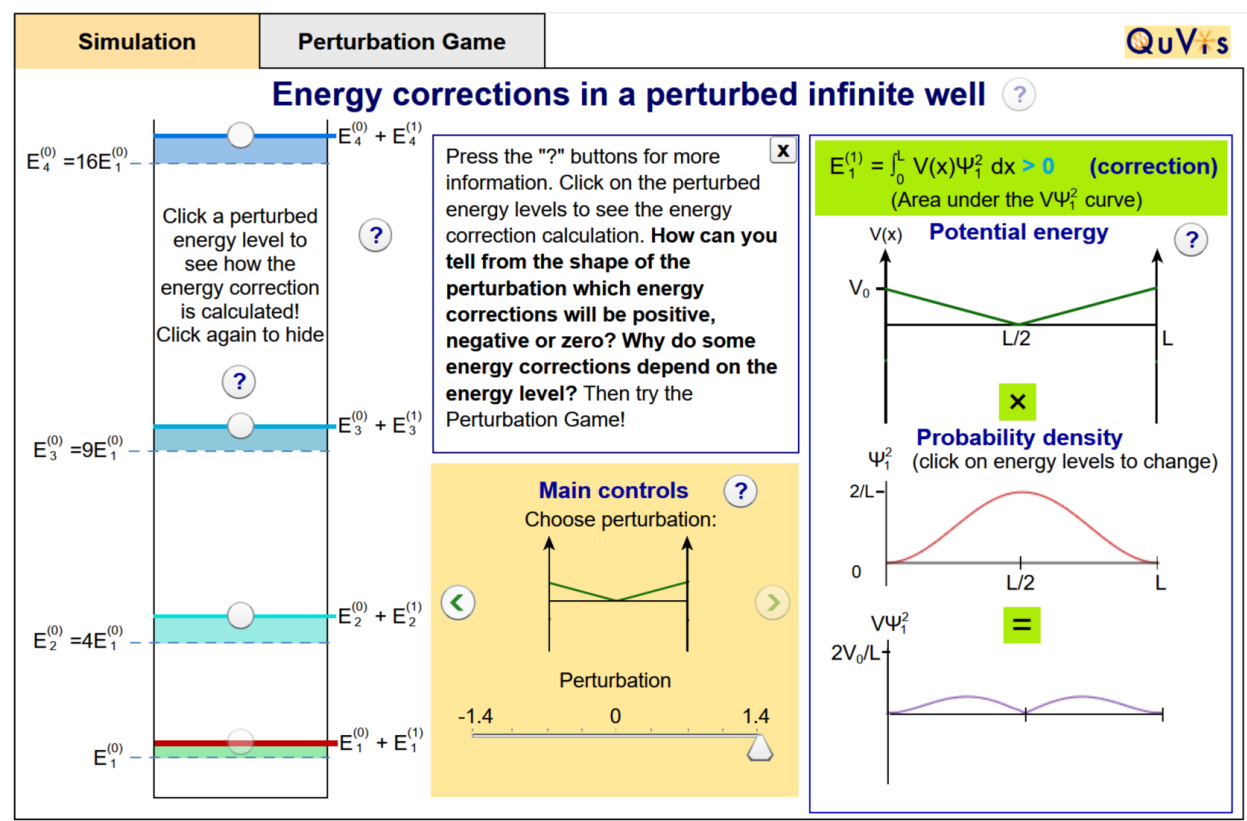

FIG. 5. A screen shot of the energy corrections in a perturbed infinite well simulation with the setup that students use for task E of the activity.

sketching does not include a physics context, so that students can focus on the properties of the visual representation without needing to additionally link the representation to domain knowledge. Thus, the task is aimed at helping students understand the properties of the representational system.

Task F in Fig. 4 asks students to sketch the product graph of two functions without reference to a physics context, and thus focuses on sketching to understand the properties of a graph of the product of two functions. Students need to consider the shape of the product curve (here that the product of two linear functions is a quadratic function), its sign, the locations of minima and maxima, and axis labels and values. This sketching task intentionally uses functions that are not depicted in the simulation, so that students must apply the methods illustrated in the simulation in this new case.

\section{Sketching to reason}

The final two tasks in Fig. 4 (tasks $\mathrm{G}$ and $\mathrm{H}$ ) involve sketching to reason with and through the representations. This is one of the core practices of science [1-3] and hence one of the key reasons it has been suggested that sketching should be integrated into science education (e.g., Ref. [53]). Sketching to reason can involve making a sketch based on underlying physics principles, or making a sketch to draw inferences and justify claims. When sketching to reason, the sketch can also be transformed to another representation to justify a claim or to assess the correctness of a statement in scientific discourse (e.g., Ref. [87]). Success in these reasoning tasks builds upon many of the aspects of visual learning that were practiced in the earlier sketching tasks and consequently are placed after these tasks.
Tasks $\mathrm{G}$ and $\mathrm{H}$ in Fig. 4 both ask students to sketch to reason. Task G (i) leverages the affordance of the simulation to quickly explore a large parameter space to discover relations. This task aims for students to explore the perturbations in the simulation to deduce that perturbations with odd symmetry will give zero energy corrections for all energy eigenfunctions, as the product curve $V\left|\psi_{n}\right|^{2}$ will be odd for this case. Thus, the task aims for students to use the concrete examples in the simulation to deduce a general principle about the energy corrections.

Task G (ii) then asks students to sketch a perturbation different to the ones shown in the simulation that would have zero energy corrections for all energy eigenfunctions. This task aims for students to reason using the abstract principle they have found in the previous part. Sketching is particularly well suited to this task, as it requires students to make their ideas concrete in terms of sketching a particular curve. Asking students to generate a different sketch to the ones shown aims to promote semantic reasoning based on the underlying meaning of the graphs in the simulation and their relation to the energy correction.

Task H (see Fig. 4) uses student dialogue to confront common incorrect ideas used by students. This technique is commonly used in the University of Washington Tutorials in Introductory Physics [26]. Often there are one or more incorrect statements and students are asked to determine which statement (if any) is correct and to explain their reasoning. The dialogues in these tasks can focus on concepts, on representations, and/or on procedure. Task $\mathrm{H}$ focuses on procedure through visual representations.

In Task H, two statements are provided, the first of which voices a common incorrect idea, namely, that the energy 
correction depends only on the average value of the perturbation. The second statement draws attention to the equation for the energy correction and correctly describes the process for solving the problem. Both statements are visual in their description but only use text and equations. The task asks students to sketch in order to determine which statement is correct. It provides three blank axes for students to use, cueing the sketching of three graphs (of the perturbation, probability density, and product graph) as done previously in the activity. After sketching to determine which statement is correct, students are asked to correct the incorrect statement. This requires attending to particular features of their sketches and transforming their sketches into verbal and mathematical descriptions to compare with the given student statements. Thus, the sketch in this case leads on to further cognitive processes, namely transforming across representations, drawing conclusions and justifying claims.

\section{Sequencing of sketching tasks}

We now return to the issue of when students sketch, and consider how theory guided the sequence of sketching tasks within each of the phases shown in Figs. 3 and 4. In common with studies of activation of prior knowledge (e.g., Refs. [74-77]), it is necessary for sketching to activate prior knowledge (task A in Fig. 3) to come before students are asked to use this knowledge to transform across representations or invent a representation. The IPFL approach by Schwartz et al. $[68,69]$ does not specify the order of the transformation and invention tasks B to D in Fig. 3. The ordering of these tasks was not determined by theory, but rather by the particular physics and representational context. In this case, the transformation from symbolic to twodimensional visual representations is an important scaffold to prepare students for the invention of a three-dimensional visual representation. In line with the IPFL approach, it is necessary to have students invent a representation in phase 1 of the activity before seeing a canonical representation in phase 2 with simulation support.

Regarding the sequence of tasks in phase 2 of the activity shown in Fig. 4, it is reasonable to start with observational sketching to ensure that students carefully observe the representations they see in the simulation. This task aims to enhance student success in the later tasks, when they explore the simulation to deduce general principles about the energy corrections and engage in more challenging sketching tasks. The sequencing of the sketching tasks to understand the properties of a representation and to reason are underpinned by considering representational learning as a two-stage process: Students first need to master the properties of canonical representations before using them flexibly to reason and justify claims [88]. Separating these two stages can be argued to promote mastery of visual representations and representational competence. This progression in the roles of sketching mirrors a progression in representational competence and in terms of expertlike use of representations [3] (see Sec. I), albeit one dramatically shortened in timescale.

\section{ANALYSIS OF STUDENTS' RESPONSES}

In this section we present our analysis of students' responses to these sketching to learn activities at two institutions [the University of St Andrews (StA), UK and California State University Fullerton (CSUF), USA] with different student populations and quantum mechanics curricula. As we followed a DBR approach, we analyze student work (and present examples) and include examples of iteration by addressing modifications made to the tasks based on student responses. The aim of this analysis was to assess whether this designed curriculum with the scaffolding put into place (in terms of the activity, peer and instructor support) afforded students opportunities to succeed at the sketching tasks, i.e., to assess the success of our design and look for evidence of when it needed refinement.

After discussing the methodology in Sec. VA, this section is organized with part VB discussing the three roles of sketching without simulation support from Sec. IV $\mathrm{A}$ and part $\mathrm{V} \mathrm{C}$ the three roles of sketching with simulation support from Sec. IV B. Because of the limited variability in students' sketches for the roles of activating prior knowledge and to observe, the discussion of these roles is quite brief. Roles with substantial variability in students' sketches (especially sketching to invent) are discussed in greater detail.

\section{A. Methodology}

At StA, the fieldwork was conducted by administering the simulation-tutorial activities in two junior-level core quantum mechanics courses following a wave mechanics instructional approach. StA is a selective, researchintensive institution in the UK. At CSUF, the activities were administered in a third-year introduction to quantum mechanics course (time development) and a senior-level quantum mechanics course (perturbation theory), both following a spins-first instructional approach [89]. CSUF is a large, teaching-intensive, Hispanic serving institution in southern California, USA. Two of the authors (A. K. and G. P.) were instructors of these courses. Class sizes ranged from 70 to 100 students at StA and 25 to 35 students at CSUF.

At both institutions, students started the activities in a class period as collaborative learning activities with the instructor (and a second person at StA) acting as facilitator, and then completed them as homework. They typically completed the first phase without simulation support and roughly half of the second phase with simulation support during the class. Instructors walked around the room and interacted with students as they worked through the activity in small groups, asking questions to promote discussion 
and support student thinking. At StA, the activities were in a lecture hall, but with a layout allowing all students to be reached by the instructor. At CSUF, the classroom tables were rearranged so students could work in groups of four and the instructor had the opportunity to have multiple interactions with each group. We draw on our experiences designing and administering these activities over three years across the two institutions for time development and perturbation theory respectively. Unless otherwise stated, examples and analysis below were drawn from the most recent version of each activity, with $N=102$ (78 StA and 24 CSUF students) and $N=61$ (44 StA and 17 CSUF students) for time development and perturbation theory respectively. Earlier iterations are only referenced to illustrate how changes to activity design (such as task wording) seemed to influence student responses.

The most important aspect of our analysis was students' sketches. Unfortunately, there is no universally agreed way to code sketches in the research literature. Consequently, through discussions among all authors, we developed multiple fine-grained approaches to provide evidence about whether each task fulfilled its specific purpose rather than apply a single coding approach throughout. Coding approaches were matched to sketching roles as shown in the two left-hand columns of Table I. The approaches were operationalized in terms of features of interest for the sketching tasks as shown in the two right-hand columns of Table I; these features are described in more detail in each of the subsections below. It should be noted that these features of interest seemed most salient in terms of the coding approaches, but we do not suggest that they are necessary or sufficient for the coding of these sketches.
As required, codes could address both the content of the sketch (e.g., the accuracy of the shape of a curve or particular features such as symmetry or adding arrows to depict time evolution) as well as standard representational requirements (e.g., axis labels and values). Therefore, for tasks such as those intended to activate prior knowledge, our focus was on accuracy in terms of content and form, while in other cases such as sketching to invent, the focus was instead on the types of sketches generated and what features were represented.

For the invention task sketches (task D), the coding was developed inductively and iteratively. Sketches were first grouped by how they utilized different axes and the sketches made previously. Then through discussion by all the authors we refined the coding to the four higher-level categories shown in Fig. 7. Subsequently, we revisited all sketches to ensure that they did fit into these categories. All sketches were coded for the features of interest in Table I initially by a first coder, and then all sketches were double checked by a second coder blind to the first person's scores. Both coders were paper authors (A. K. and G. P.). Interrater reliability in terms of percent agreement was high throughout, ranging from $88 \%$ to $100 \%$ for axis labels and values (median 97\%), $89 \%$ to $100 \%$ for the shapes of the curves (median 98\%), 92\% for the symmetry of the curve in task G, and $95 \%$ to $99 \%$ for other features (median $97 \%$ ) such as the sense of direction in task B. Interrater reliability was also calculated using Cohen's kappa. The values of kappa ranged from 0.71 to 1.0 across all of the categories, implying satisfactory to perfect agreement. These high reliability values reflect the fact that the coding scheme was sufficiently explicit that it did not require substantial

TABLE I. The two left-hand columns give an overview of the approaches used to code students' responses that were developed to align with the intended roles of sketching. The two right-hand columns show how these approaches were operationalized in terms of features of interest for the specific sketching tasks from Figs. 3 and 4. More details on these features are given in Secs. V B and V C.

\begin{tabular}{|c|c|c|c|}
\hline Sketching role & Coding approach & Tasks & Features of interest \\
\hline To activate prior knowledge & $\begin{array}{l}\text { Domain accuracy; completeness in terms } \\
\text { of standard representational requirements }\end{array}$ & A & $\begin{array}{l}\text { Shape of the curve } \\
\text { Axis labels }\end{array}$ \\
\hline $\begin{array}{l}\text { To transform across } \\
\text { representations }\end{array}$ & $\begin{array}{l}\text { Inclusion of particular features in the } \\
\text { transformed representation }\end{array}$ & $\mathrm{B}, \mathrm{C}$ & $\begin{array}{l}\text { Circular sense of rotation (B) } \\
\text { Radius value (B) } \\
\text { Values at given points (B) } \\
\text { Axis labels (C) }\end{array}$ \\
\hline To invent representations & $\begin{array}{l}\text { How previous sketches were redesigned, } \\
\text { combined and extended; how particular } \\
\text { challenges in depiction were solved }\end{array}$ & $\mathrm{D}$ & $\begin{array}{l}\text { Axes used } \\
\text { How time evolution depicted }\end{array}$ \\
\hline To observe & $\begin{array}{l}\text { Domain accuracy; completeness in terms } \\
\text { of standard representational requirements }\end{array}$ & $\mathrm{E}$ & $\begin{array}{l}\text { Shapes of the curves } \\
\text { Axis labels }\end{array}$ \\
\hline $\begin{array}{l}\text { To understand the properties } \\
\text { of a representation }\end{array}$ & Accuracy in terms of particular features & $\mathrm{F}$ & Shape of the curve \\
\hline To reason & $\begin{array}{l}\text { Domain accuracy; attending to the appropriate } \\
\text { features of the sketches to reason; consistency } \\
\text { between sketch and reasoning }\end{array}$ & $\mathrm{G}, \mathrm{H}$ & $\begin{array}{l}\text { Odd symmetry }(\mathrm{G}) \\
\text { Shape of the curve }(\mathrm{H}) \\
\text { Area under the curve }(\mathrm{H})\end{array}$ \\
\hline
\end{tabular}


subjective judgement. Therefore, the first coder's scores were taken throughout.

\section{B. Findings: Sketching to prepare for learning with the simulations}

This section describes student responses to the sketching tasks A to D (prior to working with the simulation) of the time development of infinite well quantum states simulation-tutorial activity (see Fig. 3). In this and the following section (Sec. V C), we report percentages for the features of interest for both institutions combined. Results for the two institutions were markedly similar, with the same trends observed across the different student populations.

\section{Sketching to activate prior knowledge}

Task A of the time dependence activity asked students to sketch to activate prior knowledge of the ground state energy eigenfunction $\psi_{1}(x)$ at time $t=0$ (see Fig. 3). This is a graph that is familiar to students and that they will likely have sketched before, and therefore we expected students to have no difficulty answering this task.

Students completed this task quickly with very little instructor intervention. Given the goal of activating prior knowledge, we coded students sketches in terms of their accuracy (the shape of the curve), and their completeness in including axis labels and values. Students sketches almost always had the correct shape (95\% correct), and included at least one value on the $x$ axis such as " 0 ," " $L / 2$," and " $L$ " (98\%). The majority of responses labeled the horizontal axis " $x$ " (78\%) and the vertical axis " $\psi$ " or " $\psi_{1}$ " (67\%). There was little variation in students' sketches to this task. The main variation was whether students chose to also include the potential energy well in their sketch, with 35\% of sketches doing so. This limited variation in sketching a familiar representation was in contrast to sketches of unfamiliar representations, where there were often large variations in students' sketches (see Secs. V B 2 and V B 3).

In summary, this task worked well in terms of activating relevant prior knowledge, as students were able to complete the sketching task quickly and successfully with minimal support.

\section{Sketching to transform across representations}

Tasks $\mathrm{B}$ and $\mathrm{C}$ of the time dependence activity required students to transform from symbolic to visual and from visual to text representations (see Fig. 3). Task B asked students to use a given formula to sketch the time evolution of the ground state energy eigenfunction at a particular point (giving a circle in the complex plane), and task $\mathrm{C}$ asked students to plot the full eigenfunction at a particular time where it is wholly complex [giving $i \psi_{1}(x)$ ]. Task B also asked students to describe the time evolution in words.

From our experience in the classroom, we found that this set of tasks was challenging for students and typically took
20 min for students to complete. For the coding of students' sketches transforming symbolic to visual representations, we were more interested whether or not particular features were included than in overall correctness given their limited experience at this point in the activity. Students' sketches to task B were coded for the features of direction of rotation, circle of constant radius, and values of the function at the four times provided. Students' text explanations to task B were coded for inclusion of these same features. Task C was coded for whether the student had sketched the entire function or only a single point, and whether they had correctly identified the imaginary axis.

On task B, almost all student sketches (92\%) correctly sketched the shape of a circle with a constant radius, but attention to other features (direction of rotation, radius value, and values at given points) was more variable. We considered sketches with these features to be high quality and sketches without these features to be low quality. Two example sketches are shown in Fig. 6. Sketch (a) is a lowquality sketch containing none of the additional features, whereas sketch (b) is a high-quality sketch including all three features. One of the benefits of sketching is to force students to make decisions about different aspects of the quantity being sketched. The high-quality sketch illustrates that the student has thought about and made decisions regarding the direction of rotation, the radius value, and the value of the function at different times. In contrast, the lowquality sketch only suggests that the student evaluated the function at four points, indicated by the tick marks located along each axis. However, there is no information about the directionality, the radius value, and where along this path is time $t=0$. While showing the sense of rotation was quite common ( $80 \%$ of sketches), only $60 \%$ of sketches included a radius value (and $46 \%$ a correct radius value), and $29 \%$ of sketches showed the phases or times at the four points in a variety of correct and incorrect formats.

Task B also asked students to describe the time evolution in words. This required a transformation from a visual (a) low-quality sketch

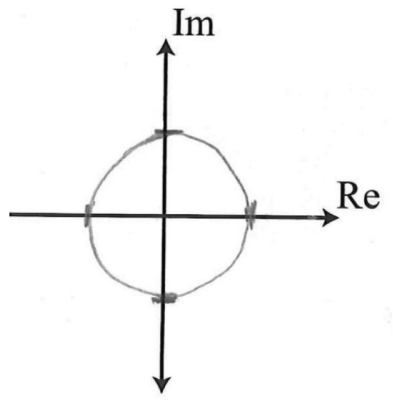

(b) high-quality sketch

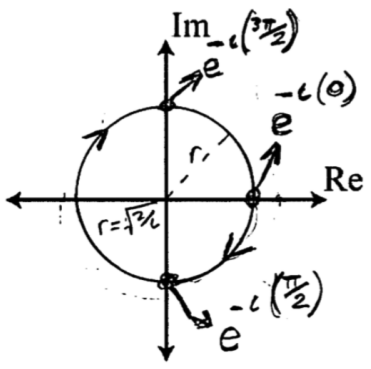

FIG. 6. Two example student sketches to task B. Sketch (a) shows a low-quality sketch that contains very little information. Sketch (b) is high quality in that it contains additional features including the direction of rotation, the radius of the circle, and the values of the function at different points. 
representation into words. One-fifth of students (21\%) wrote a complete description mentioning the constant radius and direction of motion (although very few identified the value of the radius). Most students wrote a partial description of the time evolution (58\%). These partial descriptions were missing at least one of the three features mentioned above. A further $20 \%$ of students did not write anything in response to this task.

Task $\mathrm{C}$ asked students to sketch the wave function for all positions at a different time, one where the wave function is entirely imaginary, which is a representation students may not have seen previously. This is again an instance of sketching to transform from symbolic to visual representations. We found that $75 \%$ of the students were able to sketch the wave function and identify the vertical axis as imaginary. A further $15 \%$ of the students reproduced the same type of sketch from task B that only displays the value of the function at a single point, rather than the function for all $x$, which is an important bridge to the invention in the next task.

In summary, the transformation tasks were challenging for students, but most students included at least some of the relevant features. While all students completed the sketching tasks, there were a number of blank responses for the transformation to text. This may indicate that students found the text description of a sketch either more challenging or perceived it as less worthwhile compared with transforming the symbolic to visual representations. Additional scaffolding to support high-quality sketches and especially text descriptions (such as prompts to check that all information has been included) may be useful for future iterations of the activities to increase success in transforming across representations.

\section{Sketching to invent representations}

Task D from the time-dependence activity asked students to consider how they would visualize the time evolution of the entire function using a three-dimensional representation (see Fig. 3). The sequencing of tasks provided students with previous sketches made of the entire wave function at two different fixed times and the wave function at a fixed point as a function of time. The invention task required students to synthesize and extend these previous sketches (requiring both an understanding of the individual sketches as well as the relations between them), and was thus expected to be a challenging task. In this invention task, students had to solve two problems: how to add a third axis to the sketches they had made so far with only two axes each, and how to depict the time evolution given that there are four parameters in total (the real and imaginary parts of $\psi, x$, and $t$ ) but only three axes. Given the aims of the invention task, we coded students' sketches in terms of their form and content, focusing on how the problems of three axes and depicting time evolution were solved, and how previous sketches were synthesized and extended. We did not code for correctness as this was of less interest.
In total, $69 \%$ of responses to task D included a sketch; $19 \%$ of sketches only included empty labeled axes and are not discussed further. Figure 7 shows examples of sketches seen in response to this task that illustrate the different ways that previous sketches were extended, redesigned, and combined. The figure illustrates the wide range of invented representations, of forms that are rarely seen in textbooks. Students solved the problem of the three axes mostly using the complex plane $(\operatorname{Re}$ and $\operatorname{Im})$ and $x$. Other solutions included the complex plane and $t$ [sketch (c)], or only one component of the complex plane, $x$ and $t$ [the left and middle sketches of (e)]. Students solved the problem of depicting the time evolution in multiple ways: they included a time axis [as for (c) and the left and middle graphs of (e)], overlaid multiple snapshots at different times [as for (e), the top graph of (f) and (g)], included arrows showing the sense of motion [as for the right graph of (e) and (f)], or added text descriptions of the motion [as for (a) and (g)]. Or they failed to depict time evolution at all [(b) and (d)].

We now discuss the sketches in Fig. 7 in terms of synthesizing and extending previous sketches. Figures 7(a) and 7(b) show sketches that extended the circle 2D representation from Task B to create a 3D depiction by sketching a sphere (the text states "a spinning sphere") or a cylinder. These are 3D representations familiar to students from other physics phenomena. However, these depictions are missing the visual representation of the wave function. They seem to only use the sketch from task B in terms of its surface features (the form of a circle) rather than focusing on the underlying meaning (the wave function at a fixed point in the complex plane). $7 \%$ of sketches fell into this category.

Figures 7(c) and 7(d) each accurately represent the wave function, but neither of them completely answer the task. Figure 7(c) is a redesigned version of the sketch commonly drawn for task B, with the time added as an additional dimension instead of indicated with points on the circle. This sketch is accurate, however, it does not include the wave function for all positions. $11 \%$ of sketches were redesigned versions of a previous sketch; more common was to redesign the sketch to task $\mathrm{A}$ or to task $\mathrm{C}$ to include a third axes, so that axes included both the real and imaginary parts of $\psi$.

Figure 7(d) combines the two sketches from tasks A and $\mathrm{C}$ into a single sketch. This sketch is also accurate, however, it does not include the time evolution. $10 \%$ of sketches fell into this category, with some sketches instead combining the sketches from tasks A and B. The sketches (c) and (d) are more advanced than sketches (a) and (b) in that they consider the meaning of the previous sketches in terms of the wave function at a fixed position or a fixed time, but are missing one aspect of the full 3D depiction.

The remaining figures (e), (f), and (g) all show threedimensional representations that combine and extend 
Extending the form (rather than the content) of a previous sketch

(a)

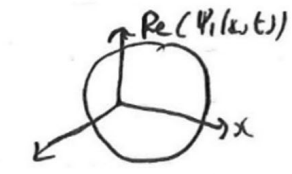

$$
\lim \left(\psi_{1}(x, t)\right) \text { a spining sphere }
$$

Redesign of a previous sketch

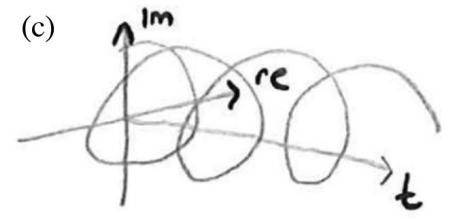

(b)

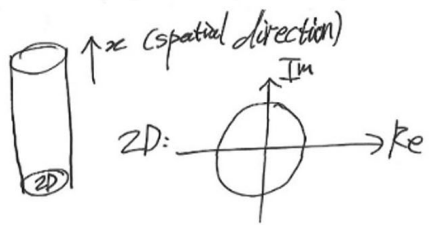

\section{Combining previous sketches}

(d)

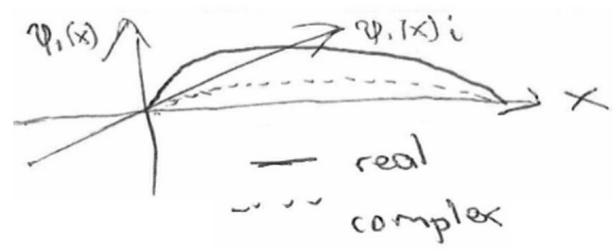

Combining and extending previous sketches

(e)

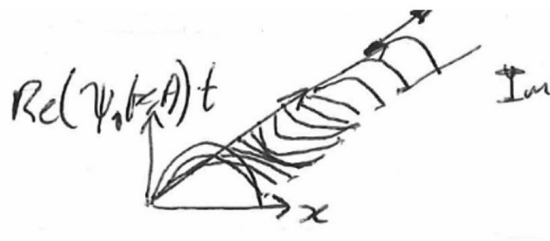

(f)

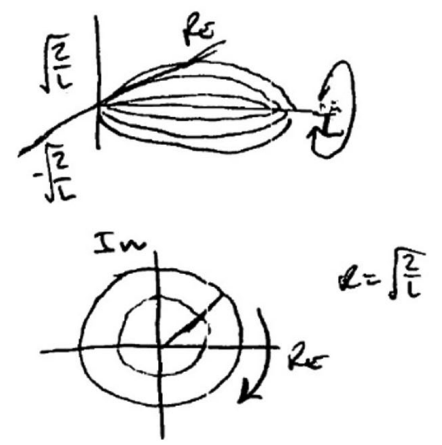

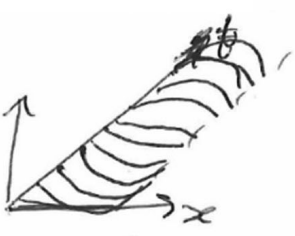

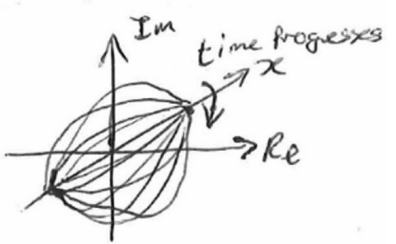

$(\mathrm{g})$

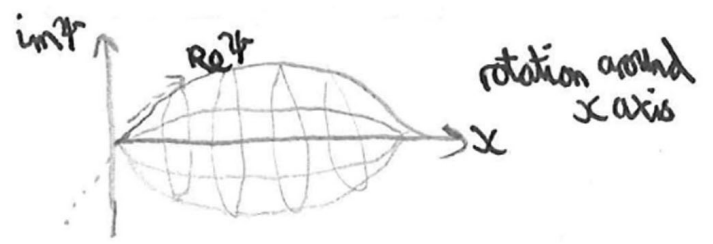

FIG. 7. Examples of students' sketches illustrating the different ways that previous sketches were extended, redesigned, and combined in the invention task (task D in Fig. 3).

previous sketches, e.g., by depicting two-dimensional projections, the time evolution as well as a 3D graph. In total, $50 \%$ of sketches fell into this category. Sketches (e), (f), and (g) utilize two different approaches to progress from two to three dimensions. In sketch (e), the student has separately drawn progressions in time for the real and imaginary components (that have a correct $90^{\circ}$ phase difference). This student has also succeeded in combining the individual graphs of the real and imaginary parts into a single 3D depiction (albeit with a shifted origin).

Figures (f) and (g) show a different path towards a 3D representation, that extends the complex plane graph generated in task B of $\psi(L / 2, t)$ to consider the time evolution at other fixed positions. The value of $\psi(x)$ determines the radius of the circle and therefore the radius changes with position. Sketch 7(f) shows an intermediate stepping stone towards a $3 \mathrm{D}$ representation, by extending the circle drawn in task B to two concentric circles, each depicting the time evolution of the wave function at a different position as well as a full 3D representation. Sketch $7(\mathrm{~g})$ shows the full time evolution as well as four circles showing the circular projection of the time evolution at four positions.

We were impressed by the wealth and quality of students' invented representations. While their attempts were not always fully successful, students solved the challenges inherent in the invention task via a range of different approaches, and built on previous sketches in multiple ways by redesigning, combining, and extending them. 


\section{Findings: Sketching while learning with the simulations}

This section describes student responses to the sketching tasks $\mathrm{E}$ to $\mathrm{H}$ (with simulation support) of the energy corrections in a perturbed infinite well simulation-tutorial activity (see Fig. 4).

\section{Sketching to observe}

Task E of the perturbation theory activity (see Fig. 4) asked students to sketch the energy level diagram and graphs they see in the simulation (see Fig. 5) for one of the given perturbations and the ground state. No elaboration or transformation of the graphs and diagrams students see in the simulation was required for these sketches. The aims of these sketches are for students to closely observe the representations shown and their features. Given these goals, we coded students' sketches in terms of their correctness and their completeness in including axis labels and relevant features.

Students completed this task without needing instructor intervention, and all students made the four sketches required for this task. Students' sketches for this task were almost always correct ( $\geq 95 \%$ for the shapes of the curves for the three graphs), and included labels ( $\geq 89 \%$ for the three graphs and $\geq 87 \%$ for the energy level diagram) and relevant features $(97 \%$ sketched the unperturbed and perturbed energy levels). Thus, the sketching task was effective in its aim of getting students to attend to features of the representations shown. The high percentage of correct answers is not surprising given that students were directly sketching what they see. Thus, observational sketching is not a challenging task.

In a previous version of the activity, we did not include additional scaffolding of three empty sets of axes for the three right-hand graphs in Fig. 5, and task E had been phrased as follows: "How can you see the energy correction of the ground state and its sign for a given perturbation (i) in the left-hand energy level diagram in the simulation (ii) in the bottom-right graph in the simulation? Explain both using sketches." We found that this version of the task did not work well for part (ii), in that the majority of students (53\%, $N=89$ for this earlier iteration) only sketched the product curve $V\left|\psi_{n}\right|^{2}$, not the graphs of the perturbation $V$ and the probability density $\left|\psi_{n}\right|^{2}$. However, the aim was for students to observe all three graphs closely, in order to link them together in terms of the graphs of $V$ and $\left|\psi_{n}\right|^{2}$ being the cause and the product graph $V\left|\psi_{n}\right|^{2}$ the effect. We therefore rephrased this task to explicitly ask for sketches of $V,\left|\psi_{1}\right|^{2}$ and the product curve $V\left|\psi_{1}\right|^{2}$ shown in the simulation, and added three empty sets of axes below the task text (not shown in Fig. 4). With these changes incorporated, all students sketched the three individual graphs in response to this task.

In summary, this latest version of the task worked well in getting students to closely observe the representations shown in the simulation, as students' sketches were complete and correct with minimal support needed beyond the simulation. The changes to the task text and the additional scaffolding in terms of empty axes were effective in ensuring that students sketched all of the visual representations shown in the simulation.

\section{Sketching to understand the properties of a visual representation}

Task F of the perturbation theory activity asked students to sketch the product of two graphs without reference to underlying physics context (see Fig. 4). The aim was to separate out mastering the ability to create a product graph and reasoning through this graph about energy corrections.

This task had previously been part of a formative assessment given after an earlier version of this activity, where we had found student difficulties with sketching a product graph. It was common (30\% of responses, $N=63$ assessments for this earlier iteration) for students to sum the two curves instead of multiplying them, leading to a horizontal line along the $x$ axis. This error is not surprising given that the graphical addition of two curves is a common technique taught in introductory physics, e.g., for the superposition of waves on a string. By moving this task from an assessment to the activity, we provided students with the opportunity to practice while having the simulation, other students, and the instructor for support.

Given that we anticipated this sketching task to be challenging with common types of errors, the coding of students' sketches focused on the shape of the product curve and how this related to addition and multiplication of the two given graphs. Excepting a small number of responses (5\%) with no sketch, the shapes of the product curves in students' sketches all fell into the three different types shown in Fig. 8. All three types of sketches are entirely negative, which indicates that students are multiplying the function with positive values by the function with negative values. The linear sketch (a) would be the result of adding $g(x)$ to an inverted $f(x)$. While this sketch indicates the students are taking into account that the two functions need to be multiplied so that the result will be entirely negative, the curve itself would be the result of addition, not multiplication. The sketch in (b) recognizes that the product of two linear graphs will result in a parabola, and the sketch in (c) goes one step further to consider the concavity of the resulting parabolas via the fact that the product curve consists of two piecewise parabolas. Although only graph (c) is technically correct, all three have productive elements in terms of being negative throughout and having a minimum in the middle and indicate that students are "on the right path" regarding multiplying functions graphically. While the majority $(52 \%)$ of responses to task F were fully correct, sketches (a) and (b) in Fig. 8 were also common (23\% and 20\%, respectively). 
(a)

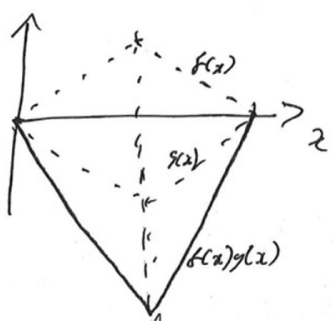

(b)

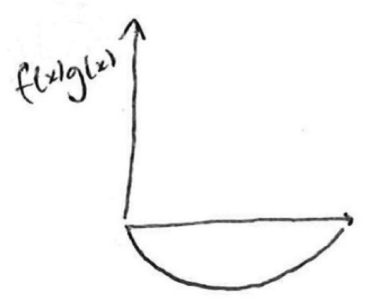

(c)

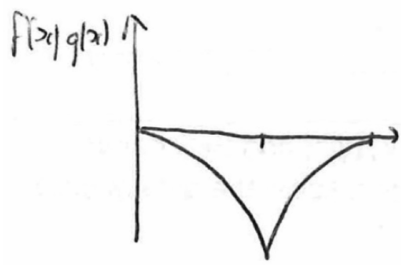

FIG. 8. The three types of sketches made in response to task F of the perturbation theory activity (see Fig. 4). Graph (c) is fully correct, but all graphs contain productive elements, including that they are negative and have a maximum in the middle of the curve.

In summary, students found the task of constructing a product graph of two curves not depicted in the simulation reasonably challenging. While many graphs were not fully correct, they all contained productive elements and including it within the activity rather than in a formative assessment did indeed increase accuracy. This indicates the importance of a sketching task focusing only on the properties of the representation without the physics content for this challenging representation.

\section{Sketching to reason}

Tasks $\mathrm{G}$ and $\mathrm{H}$ of the perturbation theory activity asked students to sketch in order to reason. Task G (i) (see Fig. 4) aimed for students to deduce from the simulation that a perturbation with odd symmetry will give zero energy corrections for all energy eigenfunctions. Task G (ii) then asked students to sketch a perturbation different from the ones shown in the simulation that would have zero energy corrections for all energy eigenfunctions. Thus, this task used sketching to reason in terms of applying a general principle to sketch a concrete curve that fulfills this principle.

Reasoning through a sketch in an expertlike fashion requires that students attend to the appropriate features of their sketch. Thus, the coding for these tasks focused on whether students' sketches were correct, included the features needed for successful reasoning, whether text made reference to these features of the sketch, and consistency between sketch and text.

Student sketches for task G (ii) were almost all correct in having odd symmetry with respect to the middle of the well (93\%), and included a range of curves including piecewise constant, piecewise linear, and trigonometric curves. Only $64 \%$ of students added text explanations, but those that did often correctly stated that their curve had odd symmetry with respect to the middle of the well $(74 \%$ of text responses). All of the text explanations were consistent with the sketch.

In a previous version of this task, the task just stated "Sketch a perturbation different to the ones shown in the simulation that would have zero energy corrections for all energy eigenfunctions." We found that some students
(11\%, $N=89$ for this earlier iteration) simply sketched the same perturbation as shown in the simulation but with a negative rather than a positive slope (as shown in the simulation if the slider is at negative values), and that only a small number of sketches included a text explanation (19\%). Thus, it had not been sufficiently clear with this earlier iteration of the task whether students' sketches were based on the principle of odd symmetry or only used a syntactic approach (based on surface features of the perturbation shown in the simulation). Given the aim of promoting semantic reasoning based on the underlying meaning of the graphs, we revised the wording to "Sketch a perturbation different to the ones shown in the simulation (this includes negative slider values) that would have zero energy corrections for all energy eigenfunctions. Explain your answer." In the revised version of the task, 93\% of sketches were different in shape to the perturbation shown in the simulation and 64\% included a text explanation. Thus, the revised version allowed us to better verify that sketches were based on an understanding of the underlying physical meaning and symmetry principles. However, further improvement in terms of encouraging text explanations would be useful.

Task H (see Fig. 4) gave students a fictive dialogue with two statements, and asked them to make sketches to decide which statements in this dialogue are correct and to correct the incorrect statements. Thus, students sketched to then reason about the given statements using their sketches. This task was made more challenging in that the perturbation is not shown in the simulation and the resulting product graph was more complicated than any the students had been asked to sketch up to this point. The version of task $\mathrm{H}$ shown in Fig. 4 has so far only been used with StA students $(N=72)$ and these results are presented here. Almost all students succeeded in sketching a product curve (99\%). Even though the product curves were not always correct $(75 \%$ fully correct), almost all curves had more area above than below the $x$ axis (92\%), which was the feature needed for successful reasoning. Almost all responses included text reasoning (93\%) and identified that statement B was correct and statement A incorrect in the fictive dialogue (92\%). Students reasoning focused on the first-order energy 
correction being the integral of $V\left|\psi_{1}\right|^{2}$ rather than the integral over $V(67 \%)$ and the perturbation having even rather than odd symmetry (44\%). Note that some students used both types of reasoning. All of the text explanations were consistent with the sketch. For future iterations, we plan to remove some of the scaffolding for this task, in terms of removing the three sets of axes or removing the correct student statement so that students need to formulate the correct argument themselves. Given that this task is close to the end of the activity, fading the scaffolding seems an important aspect of transitioning students to make appropriate representational choices themselves.

In summary, the majority of students included relevant features needed to reason successfully in their sketches and attended to these particular features in the reasoning. Even if the sketch was not fully correct, reasoning was often successful if the sketch included the correct relevant features.

\section{DISCUSSION AND CONCLUSION}

In this final section, we return to reflecting on how theory influenced the curriculum goals, the pedagogical approach, and specific choices made by the research team. We consider how these theories, our understanding of the context and students' needs, and student responses led us to refine and develop our "humble theory" of how sketching can support visual learning with interactive tutorials in quantum mechanics.

When considering the curriculum goals (what to teach), we drew on theories of representational competence and learning with multiple representations (e.g., Refs. [3,18]). Although some authors suggest that domain independent representational competence is possible $[42,43]$, we adopted a domain specific approach. We thus developed an account of representational competence in quantum mechanics, integrating theory from the learning sciences with our knowledge as practitioners.

Theory-informed predictions about what students would find easy and difficult were mostly supported in both institutions, e.g., the relative ease of observational sketching and the difficulty of transforming across representations. Interestingly however, students seemed to struggle more to transform sketches to words (or did not see the value of such tasks) than they did to construct a sketch based on a formula. This finding is not often reported in other domains, and may be due to traditional physics instruction often emphasizing mathematical formalism. Thus, a representational competence approach to quantum mechanics (and perhaps physics more generally) may need to emphasize more this transformation into words. Future work could continue to make more concrete predictions of what representational competence looks like in these domains.

When considering how to teach we combined sketching with visual learning from simulations, a relatively novel approach in science education [66]. We adopted a constructivist approach in that activity design emphasized asking students to go beyond the information that was presented to them. We leveraged the advantages of sketching as a constructive process that requires students to make their current understanding explicit in visual form, to make specific choices in order to make their ideas concrete, and to organize information to support deep processing. In general, we found that student engagement with the sketching tasks was high, as essentially all students completed the sketches suggesting that they saw value in this way to learn.

We designed a broad range of sketching tasks, including some in phase 1 prior to working with the simulation (with facilitator and peer support) and some in phase 2 with the simulation (that could be completed as homework). We identified six distinct reasons to sketch, which were sequenced in a specific way, exploring both when and why to sketch. We drew upon broad theoretical principles, for example, theories of representational competence [3] and inventing to prepare for future learning [68]. These gave us some key design requirements.

For example, the least constructive types of sketching (to activate prior knowledge and to observe) were purposefully placed at the beginning of each phase of the activities to develop students' understanding before the more cognitively demanding tasks that drew upon this understanding. Success in these tasks is important for them to fulfill their intended roles. Our findings indicate that students do indeed find these the easiest tasks to perform correctly with a very high fraction of sketches being accurate and complete. As another example, IPFL theory indicates that invention should make salient key aspects of a situation so that students can learn from subsequently presented canonical representations (in our case in the simulation). Success in this task is much less relevant for it to fulfill its intended role.

However, the sequence of tasks was not uniquely determined by theory, with key choices also made on the basis of our understanding of specific student difficulties. For example, the physics and representational context implied that the invention sketching task required scaffolding in the form of prior transformation tasks. Moreover, both practitioner knowledge and iterative design following a DBR approach were needed to refine the activities. The refinement aimed to optimize the sketching tasks in terms of their roles, e.g., via improvements to task wording and extra scaffolding. Given the link between high-quality sketches and learning (e.g., Refs. [52,63]), additional support that enables students to create high-quality sketches could benefit students in future iterations of the activities (e.g., via prompts to check that prior knowledge has been considered and all information has been included in the sketch).

The key message of this research is that the design and sequencing of sketching tasks needs to be carefully matched to the pedagogical rationale. There is not only one time and one reason to sketch. In this respect, we believe that the outcomes of this research show how these 
described sketching tasks act as design principles [40]. For example, Cobb et al. [41] suggested that "the claim that invented representations are good for mathematics and science learning probably has some merit, but it specifies neither the circumstances in which these representations might be of value nor the learning processes involved and the manner in which they are supported." Our design principle following the research is now specific, and is that asking students to invent their own representation of the time evolution of an energy eigenfunction prior to working with the simulation has specific value, draws upon students' meta-representational competence as well as their understandings of quantum mechanics and benefits from collaboration with other students and earlier scaffolding.

In keeping with a DBR approach, we will continue to refine our work to inform theory and improve our practice. For example, these activities did not include reflective sketching after completing work with the simulation. Yet this is not because we believe it is unproductive, as it may well help students remember and prompt further metacognitive insight. Rather, these activities did not have time to include it, and so we will explore it in future in other activities. We also do not claim that the six sketching tasks described here present a sufficient set of sketching tasks for the visual learning of quantum mechanics. For example, students could benefit from revising their drawings once they have interacted with simulations in the ways described by $\mathrm{Wu}$ and Rau [66] and considered by Chi [51] as interactive (more cognitively demanding and potentially even more beneficial than constructive tasks). Moreover, we found that sketching implemented for one specific role can have further value in other areas. For example, sketching is known to facilitate dialogue and discussion between peers and with instructors [80]. In our work, we found that sketches allow instructors to very quickly assess how well students were progressing. Instructors could thus provide targeted feedback if necessary, helping students overcome misunderstandings or stretching them to see new perspectives. Similarly, sketching can also play a role in summative as well as formative assessment of quantum mechanics understanding (e.g., Refs. [53,90]). Our approach suggests rather than looking for the single "best" way to sketch we should elucidate all the many reasons to sketch (as well as considering when not to do so).

In designing, implementing, and evaluating sketching tasks around interactive tutorials, we found that students could develop their representational competence in quantum mechanics, becoming increasingly able to interpret and construct visual representations central to the field. They could also sometimes transform representations from one form to another, although this (as theory suggests) still remained difficult. As we argued in the introduction, expert physicists sketch for many reasons; our research suggests so too should students learning quantum mechanics. The diversity of roles sketching can play in developing students' representational competence is evident given how many theory-based activities we developed in only two tutorial topics. Future work can design and develop further sketching activities in other topics, and then evidence whether and how students' understanding is transformed if a whole curriculum adopts this approach.

\section{ACKNOWLEDGMENTS}

The authors would like to thank all students that participated in the evaluation studies and provided feedback on the materials. We thank Michael Loverude for helpful comments on the draft manuscript. We thank the University of St Andrews for funding the development of simulations.
[1] B. Latour, Drawing things together, in Representation in Scientific Practice, edited by M. Lynch and S. Woolgar (MIT Press, Cambridge, MA 1990).

[2] D. C. Gooding, Cognition, construction and culture: Visual theories in the sciences, J. Cogn. Cult. 4, 551 (2004).

[3] R. Kozma and J. Russell, Students becoming chemists: Developing representational competence, in Visualization in Science Education, edited by J. K. Gilbert (Springer, New York, 2005), p. 121.

[4] K. L. Halverson and P. Friedrichsen, Learning tree thinking: Developing a new framework of representational competence, in Multiple Representations in Biological Education, edited by D.F. Treagust and C.-Y. Tsui (Springer, New York, 2013), p. 185.
[5] S. Nitz, S. E. Ainsworth, C. Nerdel, and H. Prechtl, Do student perceptions of teaching predict the development of representational competence and biological knowledge?, Learn. Instr. 31, 13 (2014).

[6] K. Nichols, R. Gillies, and D. Kleiss, A professional learning model that cultivates primary science classrooms' representational profiles, Int. J. Educ. Res. 76, 12 (2016).

[7] A. A. Nistal, W. Van Dooren, G. Clarebout, J. Elen, and L. Verschaffel, Conceptualising, investigating and stimulating representational flexibility in mathematical problem solving and learning: A critical review, ZDM Int. J. Math. Educ. 41, 627 (2009).

[8] D. Huinker, Representational competence: a renewed focus for classroom practice in mathematics, Wisconsin Teacher 
Math. 67, 4 (2015), http://www.wismath.org/resources/ Documents/DHuinker\%20article.pdf.

[9] P. B. Kohl and N. D. Finkelstein, Patterns of multiple representation use by experts and novices during physics problem solving, Phys. Rev. ST Phys. Educ. Res. 4, 010111 (2008).

[10] M. De Cock, Representation use and strategy choice in physics problem solving, Phys. Rev. ST Phys. Educ. Res. 8, 020117 (2012).

[11] M. Hill, M. D. Sharma, and H. Johnston, How online learning modules can improve the representational fluency and conceptual understanding of university physics students, Eur. J. Phys. 36, 045019 (2015).

[12] D. F. Treagust, R. Duit, and H. E. Fischer, Multiple Representations in Physics Education (Springer, Cham, 2017), Vol. 1.

[13] R. J. Dufresne, W. J. Gerace, and W. J. Leonard, Solving physics problems with multiple representations, Phys. Teach. 35, 270 (1997).

[14] O. Parnafes and A. Disessa, Relations between types of reasoning and computational representations, Int. J. Comput. Math. Learn. 9, 251 (2004).

[15] E. Etkina, A. Van Heuvelen, S. White-Brahmia, D. T. Brookes, M. Gentile, S. Murthy, D. Rosengrant, and A. Warren, Scientific abilities and their assessment, Phys. Rev. ST Phys. Educ. Res. 2, 020103 (2006).

[16] J. Airey and C. Linder, A disciplinary discourse perspective on university science learning: Achieving fluency in a critical constellation of modes, J. Res. Sci. Teach. 46, 27 (2009).

[17] M. Hill, M. D. Sharma, J. O’Byrne, and J. Airey, Developing and evaluating a survey for representational fluency in science, Int. J. Innovation Sci. Math. Educ. 22, 22 (2014), https://openjournals.library.sydney.edu.au/index .php/CAL/article/view/7484/8465.

[18] S. Ainsworth, Deft: A conceptual framework for considering learning with multiple representations, Learn. Instr. 16, 183 (2006).

[19] M. Stieff, M. Hegarty, and G. Deslongchamps, Identifying representational competence with multi-representational displays, Cognit. Instr. 29, 123 (2011).

[20] M. Hill and M. D. Sharma, Students' representational fluency at university: A cross-sectional measure of how multiple representations are used by physics students using the representational fluency survey, Eurasia J. Math. Sci. Technol. Educ. 11, 1633 (2015).

[21] L. C. McDermott, M. L. Rosenquist, and E. H. Van Zee, Student difficulties in connecting graphs and physics: Examples from kinematics, Am. J. Phys. 55, 503 (1987).

[22] E. Gire and E. Price, Structural features of algebraic quantum notations, Phys. Rev. ST Phys. Educ. Res. 11, 020109 (2015).

[23] A. Maries, S.-Y. Lin, and C. Singh, Challenges in designing appropriate scaffolding to improve students' representational consistency: The case of a Gauss's law problem, Phys. Rev. Phys. Educ. Res. 13, 020103 (2017).

[24] L. Bollen, P. van Kampen, C. Baily, M. Kelly, and M. De Cock, Student difficulties regarding symbolic and graphical representations of vector fields, Phys. Rev. Phys. Educ. Res. 13, 020109 (2017).
[25] A. Elby, What students' learning of representations tells us about constructivism, J. Math. Behav. 19, 481 (2000).

[26] L. C. McDermott and P. S. Shaffer, Tutorials in Introductory Physics (Prentice Hall, Upper Saddle River, 2002).

[27] A. Kohnle and G. Passante, Characterizing representational learning: A combined simulation and tutorial on perturbation theory, Phys. Rev. Phys. Educ. Res. 13, 020131 (2017).

[28] G. Passante and A. Kohnle, Enhancing student visual understanding of the time evolution of quantum systems, Phys. Rev. Phys. Educ. Res. 15, 010110 (2019).

[29] J. L. Plass, B. D. Homer, and E. O. Hayward, Design factors for educationally effective animations and simulations, J. Computing Higher Educ. 21, 31 (2009).

[30] N. S. Podolefsky, K. K. Perkins, and W. K. Adams, Factors promoting engaged exploration with computer simulations, Phys. Rev. ST Phys. Educ. Res. 6, 020117 (2010).

[31] N. Rutten, W. R. Van Joolingen, and J. T. Van der Veen, The learning effects of computer simulations in science education, Comput. Educ. 58, 136 (2012).

[32] E. B. Moore, T. A. Herzog, and K. K. Perkins, Interactive simulations as implicit support for guided-inquiry, Chem. Educ. Res. Pract. 14, 257 (2013).

[33] A. Kohnle, C. Baily, A. Campbell, N. Korolkova, and M. J. Paetkau, Enhancing student learning of two-level quantum systems with interactive simulations, Am. J. Phys. 83, 560 (2015).

[34] J. J. Kaput, Technology and mathematics education, in Handbook of Teaching and Learning Mathematics (Macmillan Publishing Company, New York, 1992).

[35] J. van der Meij and T. de Jong, Supporting students' learning with multiple representations in a dynamic simulation-based learning environment, Learn. Instr. 16, 199 (2006).

[36] M. A. Rau, Conditions for the effectiveness of multiple visual representations in enhancing stem learning, Educ. Psychol. Rev. 29, 717 (2017).

[37] T. Seufert, Supporting coherence formation in learning from multiple representations, Learn. Instr. 13, 227 (2003).

[38] S. Barab and K. Squire, Design-based research: Putting a stake in the ground, J. Learn. Sci. 13, 1 (2004).

[39] A. Collins, D. Joseph, and K. Bielaczyc, Design research: Theoretical and methodological issues, J. Learn. Sci. 13, 15 (2004).

[40] T. Anderson and J. Shattuck, Design-based research: A decade of progress in education research?, Educ. Res. 41, 16 (2012).

[41] P. Cobb, J. Confrey, A. DiSessa, R. Lehrer, and L. Schauble, Design experiments in educational research, Educ. Res. 32, 9 (2003).

[42] H.-Y. Chang, Students' representational competence with drawing technology across two domains of science, Sci. Educ. 102, 1129 (2018).

[43] K. L. Daniel, C. J. Bucklin, E. A. Leone, and J. Idema, Towards a definition of representational competence, in Towards a Framework for Representational Competence in Science Education, edited by K. L. Daniel (Springer, New York, 2018), p. 3.

[44] Andrea A. diSessa, Metarepresentation: Native competence and targets for instruction, Cognit. Instr. 22, 293 (2004). 
[45] E. Marshman and C. Singh, Framework for understanding the patterns of student difficulties in quantum mechanics, Phys. Rev. ST Phys. Educ. Res. 11, 020119 (2015).

[46] C. Singh and E. Marshman, Review of student difficulties in upper-level quantum mechanics, Phys. Rev. ST Phys. Educ. Res. 11, 020117 (2015).

[47] K. Krijtenburg-Lewerissa, H. J. Pol, A. Brinkman, and W. R. Van Joolingen, Insights into teaching quantum mechanics in secondary and lower undergraduate education, Phys. Rev. Phys. Educ. Res. 13, 010109 (2017).

[48] H. G. Close, E. W. Close, and D. Donnelly, Nesting in graphical representations in physics, AIP Conf. Proc. 1513, 110 (2013).

[49] J.S. Bruner, Toward a Theory of Instruction (Norton, New York, 1966).

[50] J. Piaget, To Understand is to Invent (Grossman, New York, 1973).

[51] M. T. H. Chi, Active-constructive-interactive: A conceptual framework for differentiating learning activities, Topics Cogn. Sci. 1, 73 (2009).

[52] P. Van Meter and J. Garner, The promise and practice of learner-generated drawing: Literature review and synthesis, Educ. Psychol. Rev. 17, 285 (2005).

[53] S. Ainsworth, V. Prain, and R. Tytler, Drawing to learn in science, Science 333, 1096 (2011).

[54] P. Van Meter and C. M. Firetto, Cognitive model of drawing construction, in Learning through Visual Displays, edited by G. Schraw, M. T. McCrudden, and D. Robinson (Information Age Publishing, Charlotte, NC, 2013), p. 247.

[55] K. D. Forbus and S. Ainsworth, Editors' introduction: Sketching and cognition, Topics Cogn. Sci. 9, 864 (2017).

[56] L. Fiorella and Q. Zhang, Drawing boundary conditions for learning by drawing, Educ. Psychol. Rev. 30, 1115 (2018).

[57] S. P. W. Wu and M. A. Rau, How students learn content in science, technology, engineering, and mathematics (stem) through drawing activities, Educ. Psychol. Rev. 31, 87 (2019).

[58] M. T. H. Chi, M. Bassok, M. W. Lewis, P. Reimann, and R. Glaser, Self-explanations: How students study and use examples in learning to solve problems, Cogn. Sci. 13, 145 (1989).

[59] K. Stenning and J. Oberlander, A cognitive theory of graphical and linguistic reasoning: Logic and implementation, Cogn. Sci. 19, 97 (1995).

[60] J. Zhang and D. A. Norman, Representations in distributed cognitive tasks, Cogn. Sci. 18, 87 (1994).

[61] J. H. Larkin and H. A. Simon, Why a diagram is (sometimes) worth ten thousand words, Cogn. Sci. 11, 65 (1987).

[62] S. Ainsworth and A. T. Loizou, The effects of self-explaining when learning with text or diagrams, Cogn. Sci. 27, 669 (2003).

[63] K. Scheiter, K. Schleinschok, and S. Ainsworth, Why sketching may aid learning from science texts: Contrasting sketching with written explanations, Topics Cogn. Sci. 9, 866 (2017).

[64] J. G. Cromley, L. E. Snyder-Hogan, and U. A. LuciwDubas, Cognitive activities in complex science text and diagrams, Contemp. Educ. Psychol. 35, 59 (2010).

[65] L. Mason, M. C. Tornatora, and P. Pluchino, Do fourth graders integrate text and picture in processing and learning from an illustrated science text? evidence from eye-movement patterns, Comput. Educ. 60, 95 (2013).

[66] S. P. W. Wu and M. A. Rau, Effectiveness and efficiency of adding drawing prompts to an interactive educational technology when learning with visual representations, Learn. Instr. 55, 93 (2018).

[67] Z.H. Zhang and M. C. Linn, Can generating representations enhance learning with dynamic visualizations?, J. Res. Sci. Teach. 48, 1177 (2011).

[68] D. L. Schwartz and T. Martin, Inventing to prepare for future learning: The hidden efficiency of encouraging original student production in statistics instruction, Cognit. Instr. 22, 129 (2004).

[69] D. L. Schwartz, C. C. Chase, M. A. Oppezzo, and D. B. Chin, Practicing versus inventing with contrasting cases: The effects of telling first on learning and transfer, J. Educ. Psychol. 103, 759 (2011).

[70] S. Brahmia, A. Boudreaux, and S. E. Kanim, Developing mathematical creativity with physics invention tasks, arXiv:1602.02033.

[71] University of St Andrews QuVis Quantum Mechanics Visualization Project, Time-development of infinite well quantum states, https://www.st-andrews.ac.uk/ physics/quvis/simulations_html5/sims/TimeDevelopment/ TimeDevelopment.html (Accessed June 18, 2019).

[72] University of St Andrews QuVis Quantum Mechanics Visualization Project, Energy corrections in a perturbed infinite well, https://www.st-andrews.ac.uk/ physics/quvis/simulations_html5/sims/perturbationGame/ perturbationGame.html (Accessed June 18, 2019).

[73] See Supplemental Material at http://link.aps.org/ supplemental/10.1103/PhysRevPhysEducRes.16.020139 for the full simulation-tutorial activities described in the text.

[74] R. E. Mayer, Twenty years of research on advance organizers: Assimilation theory is still the best predictor of results, Instr. Sci. 8, 133 (1979).

[75] M. Pressley, E. Wood, V. E. Woloshyn, V. Martin, A. King, and D. Menke, Encouraging mindful use of prior knowledge: Attempting to construct explanatory answers facilitates learning, Educ. Psychol. 27, 91 (1992).

[76] M. Machiels-Bongaerts, H. G. Schmidt, and H.P. A. Boshuizen, Effects of mobilizing prior knowledge on information processing: Studies of free recall and allocation of study time, Br. J. Psychol. 84, 481 (1993).

[77] S. Wetzels, L. Kester, and J. Van Merriënboer, Use of external representations in science: prompting and reinforcing prior knowledge activation, in Use of Representations in Reasoning and Problem Solving, edited by L. Verschaffel, E. Corte, T. Jong, and J. Elen (Routledge, London, 2010), p. 225.

[78] S. Ainsworth, The functions of multiple representations, Comput. Educ. 33, 131 (1999).

[79] G. Kress, C. Jewitt, J. Ogborn, and T. Charalampos, Multimodal Teaching and Learning: The Rhetorics of the Science Classroom (Continuum, London, 2001).

[80] V. Prain and R. Tytler, Learning through constructing representations in science: A framework of representational construction affordances, Int. J. Sci. Educ. 34, 2751 (2012). 
[81] K. E. Vaughn and K. A. Rawson, When is guessing incorrectly better than studying for enhancing memory?, Psychon. Bull. Rev. 19, 899 (2012).

[82] G. Brod, M. Hasselhorn, and S. A. Bunge, When generating a prediction boosts learning: The element of surprise, Learning Instr. 55, 22 (2018).

[83] J. Wiley, Picture this! Effects of photographs, diagrams, animations, and sketching on learning and beliefs about learning from a geoscience text, Appl. Cogn. Psychol. 33, 9 (2019).

[84] M. A. Fernandes, J. D. Wammes, and M. E. Meade, The surprisingly powerful influence of drawing on memory, Curr. Dir. Psychol. Sci. 27, 302 (2018).

[85] J. L. Lemke, The literacies of science, in Crossing Borders in Literacy and Science Instruction: Perspectives on Theory and Practice, edited by E. W. Saul (Int. Reading Assoc., Newark, NJ, 2004).

[86] J. G. Cromley, T. C. Perez, S. L. Fitzhugh, N. S. Newcombe, T. W. Wills, and J. C. Tanaka, Improving students' diagram comprehension with classroom instruction, J. Exp. Educ. 81, 511 (2013).

[87] R. Kozma, E. Chin, J. Russell, and N. Marx, The roles of representations and tools in the chemistry laboratory and their implications for chemistry learning, J. Learn. Sci. 9, 105 (2000).

[88] M. A. Rau, J. E. Michaelis, and N. Fay, Connection making between multiple graphical representations: A multimethods approach for domain-specific grounding of an intelligent tutoring system for chemistry, Comput. Educ. 82, 460 (2015).

[89] H. R. Sadaghiani and J. Munteanu, Spin first instructional approach to teaching quantum mechanics in sophomore level modern physics courses, in Proceedings of the 2015 Physics Education Research Conference, College Park, MD (AIP, New York, 2015), p. 287.

[90] M. M. Cooper, L. C. Williams, and S. M. Underwood, Student understanding of intermolecular forces: A multimodal study, J. Chem. Educ. 92, 1288 (2015). 